\title{
Generalization of Konhauser Polynomials
}

\author{
Mamta Dassani, Mukesh kushwaha
}

\begin{abstract}
In this paper, we are showing study of biorthogonal polynomials associated with generalization of Laguere polynomials of Srivastava and Singhal [14]. It happens to generalized Konhauser. here we are trying to obtain the generating functions, recurrence relations, biorthogonality relations, integral representations and also bilinear and bilateral generating relations for the new class of biorthogonal system.

Keywords: Biorthogonal polynomials, Laguere polynomials, generalized Konhauser, generating functions, recurrence relations,
\end{abstract}

\section{INTRODUCTION}

The concept of two polynomials explain by Didon [1] and Deruyts [2] considered this concept in some detail. For example, given the set $\{\operatorname{Pn}(\mathrm{x})\}$ the set $\{\mathrm{Qn}(\mathrm{x})\}$ is uniquely determined and conversely over the different interval. considered this concept in some detail, and claimed that for these two polynomials. the set of polynomials in $\mathrm{x},\left\{\mathrm{P}_{\mathrm{n}}(\mathrm{x})\right\}$ and $\left\{\mathrm{Q}_{\mathrm{n}}(\mathrm{x})\right\}$, deg. $\mathrm{Q}_{\mathrm{n}}(\mathrm{x})=\mathrm{n},(\mathrm{n}=0,1,2, \ldots .$.$) are said to be$ biorthogonal with respect to distribution $\mathrm{d} \alpha$ (x) on interval [a,b] if:

$$
\int_{\mathrm{a}}^{\mathrm{b}} \mathrm{P}_{\mathrm{n}}(\mathrm{x}) \mathrm{Q}_{\mathrm{n}}(\mathrm{x}) \mathrm{da}(\mathrm{x})=0, \mathrm{~m} \neq \mathrm{n} \neq 0, \mathrm{~m}=\mathrm{n}
$$

where $\alpha(\mathrm{x})$ is a distribution function on interval (finite or infinite) with infinitely many points of increase and such

that : $\int_{\mathrm{a}}^{\mathrm{b}} \mathrm{x}^{\mathrm{n}} \mathrm{d} \alpha(\mathrm{x})<\infty$, for all $\mathrm{n}=0,1,2, \ldots$.

Not much attention was paid to the study of biorthogonal system of polynomials, till Spencer and Fano [3] encountered a pair of biorthogonal polynomials, while dealing with a problem related to the study of penetration and diffusion of X-Rays, and subsequently studies were made by Preiser [4] in ordinary differential equation of the third order and it also recommend for higer order form .Konhauser [5] , Carlitz [6] , Prabhakar and Kashyap [7] and Prabhakar \& Tomar [9] describe some results on biorthognal function suggested by the Laguerre polynomials. Rahman [8] also expressed some explicit function of unearization coefficient of the product of Jacobi polynomials ,

Revised Manuscript Received on March 10, 2020.

* Correspondence Author

Mamta Dassani*, Deptt. of Basic Science, Bundelkhand University Jhansi, India. Email: Mamtavalecha09@gmail.com

Mukesh Kushwaha, Deptt. of Mathematical Scienes \& Computer Applications, Bundelkhand University, Jhansi, India. Email: mukeshkus12785@gmail.com

(c) The Authors. Published by Blue Eyes Intelligence Engineering and Sciences Publication (BEIESP). This is an open access article under the CC BY-NC-ND license (http://creativecommons.org/licenses/by-nc-nd/4.0/)
Madhekar and Thakare [10] has work on Biorthogonal polynomials suggested by the Jacobi polynomials and AlSalam and Verma [11] also described Analogues of some biorthogonal function. Both Didon and Deruyts paid special attention to the situation in which $\mathrm{P}_{\mathrm{n}}(\mathrm{x})$ is a polynomial of degree $n$ in $x^{\mathrm{k}}$ (k fixed)

In this paper, we shall study the generalization of biorthogonal Polynomials suggested by Konhauser polynomials over the interval $(0, \infty)$ with respect to the distribution function $\mathrm{w}(\mathrm{x})=\mathrm{x}^{\alpha} \exp \left(-\mathrm{pr}^{\mathrm{r}}\right) \mathrm{dx}$ and also Obtain associated generating relations for $Y_{n}^{(\alpha)}(x, r, p, k)$ and $Z_{n}^{(\alpha)}(x, r, p, k)$. We recall the polynomials $G_{n}^{(\alpha)}(x, r, p, k)$, which are introduced by Srivastava and Singhal [14] and in attempt to provide an elegant unification of various known generalized of classical Hermite and Laguerre polynomials. These polynomials are defined by the generalized Rodrigues's formula

$G_{n}^{(\alpha)}(x, r, p, k)=x^{-k n-\alpha} \exp \left(p x^{r}\left(\frac{1}{n !}\right)\left(x^{k+1} D_{x}\right)^{n}\left\{x^{\alpha} \exp \left(-p x^{r}\right)\right\}\right.$,

where $D_{x}=d / d x$ and parameters $\alpha, k, p$ and $r$ are unrestricted in general. The explicit expansion is given as $G_{n}^{(\alpha)}(x, r, p, k)=\left(\frac{K^{n}}{n !}\right) \sum_{i=0}^{n}\left(p x^{r}\right)\left(\frac{1}{l !}\right) \sum_{j=0}^{i}(-1)^{j}\left(\begin{array}{l}i \\ j\end{array}\right)\left(\frac{r j+\alpha}{k}\right)_{n}$

It is worth mentioning here that Srivastava and Singhal [14], Chandel [1] and Srivastava, P.N. [16] also consider the polynomials

$$
\mathrm{Y}_{\mathrm{n}}^{(\alpha)}(\mathrm{x}, \mathrm{k})=\mathrm{k}^{-\mathrm{n}} \mathrm{G}_{\mathrm{n}}^{(\alpha+1)}(\mathrm{x}, 1,1, \mathrm{k})
$$
defined

Thus, we observe that (2) provides a generalization of one member of the pair of Konhauser biorthogonal polynomials. This leads us to consider pair biorthogonal polynomials, one of which is connected with (1)

\section{PRELIMINARIES}

\section{A. Generalized konhauser polynomials}

In this section, we included the different kind of relation which are pair of biorthogonal sets of polynomials see $[12,13,15]$.

$Y_{n}^{(\alpha)}(x, r, p, k)$ and $Z_{n}^{(\alpha)}(x, r, p, k)$, where $Z_{n}^{(\alpha)}(x, r, p, k)$ is a polynomial of degree $n$ in $x^{k}$, ( $k$ is fixed integer) while $Y_{n}^{(\alpha)}(x, r, p, k)$ is a polynomial of degree $n$ in, $X^{r}$, ( $r$ is fixed integer

$\mathrm{Z}_{\mathrm{n}}^{(\alpha)}(\mathrm{x}, \mathrm{r}, \mathrm{p}, \mathrm{k})=\frac{\Gamma\{(\alpha+1+\mathrm{kn}) / \mathrm{r}\}}{\mathrm{p}^{\mathrm{kn} / \mathrm{r}} \mathrm{n} !} \sum_{\mathrm{m}=0}^{\mathrm{n}}(-1)^{\mathrm{m}}\left(\begin{array}{l}\mathrm{n} \\ \mathrm{m}\end{array}\right) \frac{\mathrm{p}^{\mathrm{km} / \mathrm{r}} \mathrm{x}^{\mathrm{km}}}{\{(\alpha+1+\mathrm{km}) / \mathrm{r}\}}$ 


\section{Generalization of Konhauser Polynomials}

$\mathrm{Y}_{\mathrm{n}}^{(\alpha)}(\mathrm{x}, \mathrm{r}, \mathrm{p}, \mathrm{k})=(1 / \mathrm{n} !) \sum_{\mathrm{i}=0}^{\mathrm{n}} \frac{\mathrm{p}^{\mathrm{i}} \mathrm{x}^{\mathrm{ri}}}{\mathrm{i} !} \sum_{\mathrm{j}=0}^{\mathrm{n}}(-1)^{\mathrm{i}}\left(\begin{array}{l}\mathrm{i} \\ \mathrm{j}\end{array}\right)\left(\frac{\alpha+1+\mathrm{rj}}{\mathrm{k}}\right)_{\mathrm{n}}$

where $(\alpha+1) / r>0$ and $k / r$ is a positive integer.

\section{B. First biorthogonal relation}

The polynomials sets

$\mathrm{Y}_{\mathrm{n}}^{(\alpha)}(\mathrm{x}, \mathrm{r}, \mathrm{p}, \mathrm{k})$ and $\mathrm{Z}_{\mathrm{n}}^{(\alpha)}(\mathrm{x}, \mathrm{r}, \mathrm{p}, \mathrm{k})$ are biorthogonal

with respect to the distribution function $\mathrm{w}(\mathrm{x})=\mathrm{X}^{(\alpha)} \exp$

$\left(-\mathrm{px}^{\mathrm{r}}\right)$ over the interval $(0, \infty)$

The biorthogonal relation is given by (4)

$\int_{0}^{\infty} \mathrm{x}^{\alpha} \exp \left(-\mathrm{px}^{\mathrm{r}}\right) \mathrm{Y}_{\mathrm{m}}^{(\alpha)}(\mathrm{x}, \mathrm{r}, \mathrm{p} ; \mathrm{k}) \mathrm{z}_{\mathrm{n}}^{(\alpha)}(\mathrm{z}, \mathrm{r}, \mathrm{p} ; \mathrm{k}) \mathrm{dx}=\frac{\Gamma\{(\alpha+1+\mathrm{kn}) / \mathrm{r}\}}{\mathrm{rm} ! \mathrm{p}^{(\alpha+1+\mathrm{kn}) / \mathrm{r}}} \delta \mathrm{m}, \mathrm{n}$

where $\delta \mathrm{m}, \mathrm{n}$ is Kronecker delta and $\mathrm{k} / \mathrm{r}$ is a positive integer. We shall prove this relation later on.

C. Generating function for $\mathrm{z}_{\mathrm{N}}^{(\alpha)}(\mathrm{x}, \mathrm{r}, \mathrm{p} ; \mathrm{k})$ and $\mathrm{Y}_{\mathrm{n}}^{(\alpha)}(\mathrm{x}, \mathrm{r}, \mathrm{p} ; \mathrm{k})$

use (2), we have

$\mathrm{Z}_{\mathrm{n}}^{(\alpha)}(\mathrm{x}, \mathrm{r}, \mathrm{p} ; \mathrm{k})=\frac{\Gamma\{(\alpha+1+\mathrm{kn}) / \mathrm{r}\}}{\mathrm{p}^{\mathrm{kn} / \mathrm{r}} \mathrm{n} !} \sum_{\mathrm{m}=0}^{\mathrm{n}}(-1)^{\mathrm{m}}\left(\begin{array}{l}\mathrm{n} \\ \mathrm{m}\end{array}\right) \frac{\mathrm{p}^{\mathrm{km} / \mathrm{r}} \mathrm{x}^{\mathrm{km}}}{\Gamma\{(\alpha+1+\mathrm{km}) / \mathrm{r}\}}$

$\mathrm{Z}_{\mathrm{n}}^{(\alpha)}(\mathrm{x}, \mathrm{r}, \mathrm{p} ; \mathrm{k})=\frac{(\{\mathrm{a}+1\} / \mathrm{r}) \mathrm{q}_{\mathrm{n}}}{\mathrm{p}^{\mathrm{kn} / \mathrm{r}} \mathrm{n} !} \sum_{\mathrm{m}=0}^{\mathrm{n}}(-\mathrm{n})_{\mathrm{m}} \frac{\mathrm{p}^{\mathrm{km} / \mathrm{r}} \mathrm{x}^{\mathrm{km}}}{\mathrm{m} ! \Gamma\{(\alpha+1+\mathrm{km}) / \mathrm{r}\}}$

$Z_{n}^{(\alpha)}(x, r, p ; k)=\frac{(\{a+1\} / r) q_{n}}{p^{k n / r} n !} \sum_{m=0}^{n}(-n)_{m} \frac{\left(p^{k m / r} x^{k m}\right)^{m}}{m ! q^{a m} \prod_{s=1}^{q}[\{(\alpha+1) / r+s-1\} / q]}$

$=\frac{(\{\alpha+1\} / r) q n}{p^{k m / r} n !} F_{q}\left[-n,(\alpha+1) / r q \ldots \ldots . .\{\alpha+1+r(q-1)\} / r q ;\left(p^{q} x^{r q}\right) / q^{q}\right]$

thus, $\mathrm{Z}_{\mathrm{n}}^{(\alpha)}(\mathrm{x}, \mathrm{r}, \mathrm{p} ; \mathrm{k})$ is in the hypergeometric form.

where $\mathrm{k} / \mathrm{r}=\mathrm{q}$, a positive integer.

Now,

$\sum_{n=0}^{\infty} Z_{n}^{\alpha}(x, r, p ; q) \frac{t^{n}}{((\alpha+1) / r)_{q n}}=\sum_{n=0}^{\infty} \frac{t^{n}}{n ! p^{q n}} \sum_{m=0}^{\infty}(-1)^{m}\left(\begin{array}{l}n \\ m\end{array}\right) \frac{p^{m q} x^{k m}}{((\alpha+1) / r)_{q n}}$

$=\sum_{\mathrm{n}=0}^{\infty} \sum_{\mathrm{m}=0}^{\infty}(-1)^{\mathrm{m}+\mathrm{n}}\left(\begin{array}{l}\mathrm{n}+\mathrm{m} \\ \mathrm{n}\end{array}\right) \frac{\mathrm{t}^{\mathrm{n}+\mathrm{m}} \mathrm{x}^{\mathrm{km}}}{(\mathrm{n}+\mathrm{m}) \cdot \mathrm{p}^{\mathrm{q}(\mathrm{n}+\mathrm{m})} \prod_{s=1}^{\mathrm{q}}[(((\alpha+1) / \mathrm{r})+\mathrm{s}-1\} / \mathrm{q}]}$

$=\sum_{\mathrm{n}=0}^{\infty} \frac{(-\mathrm{t})^{\mathrm{n}}}{\mathrm{p}^{\mathrm{kn} / \mathrm{r}}} \sum_{\mathrm{n}=0}^{\infty} \frac{(-1)^{\mathrm{m}} \mathrm{x}^{\mathrm{km}} \mathrm{t}^{\mathrm{m}}}{\mathrm{m} ! \prod_{\mathrm{s}=1}^{\mathrm{q}}[\{((\alpha+1) / \mathrm{r})+\mathrm{s}-1\} / \mathrm{q}]}$

$=\exp (-t / p)^{k / r}{ }_{0} F_{q}\left[\ldots \ldots . . ;(\alpha+1) / r q \ldots \ldots . . .(\alpha+1+r(q-1)\} / r q,(-x / q)^{r q} t\right]$

which is the generating function for $Z_{n}^{\alpha}(x, r, p ; q)$.

use (1) and (2), we observe that

$Y_{n}^{\alpha}(x, r, p ; k)=k^{-n} G_{n}^{(\alpha+1)}(x, r, p ; k)$

Hence $Y_{n}^{\alpha}(x, r, p ; k)=\frac{x^{-\alpha-k n-1} \exp \left(p x^{r}\right)}{k^{n} n !}\left(x^{k+1} D\right)^{n}\left[x^{\alpha+1} \exp \left(-p x^{r}\right)\right]$

Now using the analogues result, we get a generating function for $\mathrm{Y}_{\mathrm{n}}^{\alpha}(\mathrm{x}, \mathrm{r}, \mathrm{p} ; \mathrm{k})$ as:

$\sum_{n=0}^{\infty} Y_{n}^{\alpha}(x, r, p ; k) t^{n}=(1-t)^{-(\alpha+1) / k} \exp \left[p^{r}\left\{1-(1-t)^{-r / k}\right\}\right]$

\section{Second biorthogonal relation}

We have to prove relation (4)
Consider,,

$\left.=\sum_{\mathrm{n}=0}^{\infty} \sum_{\mathrm{m}=0}^{\infty} \int_{0}^{\infty} x^{\alpha} \exp \left(-p x^{r}\right) Y_{n}^{\alpha}(x, r, p ; k) Z_{m}^{\alpha}(x, r, p ; k)\left[\frac{1}{(\{1+\alpha\} / r)}\right)_{\mathrm{qm}}\right] u^{\mathrm{m}} \mathrm{t}^{\mathrm{n}} \mathrm{dx}$

$=\int_{0}^{\infty} x^{\alpha} \exp \left(-p x^{r}\right)\left[\sum_{n=0}^{\infty} Y_{n}^{\alpha}(x, r, p ; k) t^{n}\right]\left[\sum_{n=0}^{\infty} Z_{m}^{\alpha}(x, r, p ; k)\left[\frac{1}{(\{\alpha+1\} / r)_{m}}\right] u^{m}\right] d x$

$=\int_{0}^{\infty} \mathrm{x}^{\alpha} \exp \left(-\mathrm{px}^{\mathrm{r}}\right)\left[(1-\mathrm{t})^{\{-(-1+\alpha) / k\}} \exp \left[-\mathrm{px}^{\mathrm{r}}\left\{-1-\mathrm{t}^{-\mathrm{r} / \mathrm{k}}\right\}\right]\right] \exp (-\mathrm{t} / \mathrm{p})^{k / \mathrm{r}}$

$={ }_{0} \mathrm{~F}_{\mathrm{q}}\left[\ldots .,(\alpha+1) / \mathrm{rq}, \ldots . .\{\alpha+1+\mathrm{r}(\mathrm{q}-1)\} / \mathrm{rq} ;(\mathrm{x} / \mathrm{q})^{\mathrm{m}} \mathrm{u}\right]$

[Using (4) and (5)]

$=(1-t)^{-(-1+\alpha) / k\}} \exp (-u / p)^{k / t} \sum_{m=0}^{\infty} \frac{(-u)^{m}}{((1+\alpha) / r)_{q m}} \int_{0}^{\infty} x^{\alpha+q m} \exp \left[-p^{r}\left\{(1-t)^{-r / k}\right] d x\right.$

after some simplification, we get; (9)

$\int_{0}^{\infty} \mathrm{x}^{\alpha} \exp \left(-\mathrm{px}^{\mathrm{r}}\right)\left[\sum_{\mathrm{n}=0}^{\infty} \mathrm{Y}_{\mathrm{n}}^{\alpha}(\mathrm{x}, \mathrm{r}, \mathrm{p}, \mathrm{k} \mathrm{k}) \mathrm{t}^{\mathrm{n}}\right]\left[\sum_{\mathrm{m}=0}^{\infty} \mathrm{Z}_{\mathrm{m}}^{\alpha}(\mathrm{x}, \mathrm{r}, \mathrm{p}, \mathrm{k})\left[\frac{1}{(\{\alpha+1\} / \mathrm{r})_{\mathrm{qm}}}\right] \mathrm{u}^{\mathrm{m}}\right] \mathrm{dx}=$

$=\frac{\Gamma\{(\alpha+1) / \mathrm{r}\}}{r . p^{\{(\alpha+1) / r\}}} \exp \left(\mathrm{ut} / \mathrm{p}^{\mathrm{q}}\right) \frac{\Gamma((\alpha+1) / \mathrm{r}\}}{\Gamma \mathrm{p}^{[(\alpha+1) / \mathrm{r}\}}} \sum_{\mathrm{m}=0}^{\infty}(1 / \mathrm{m} !)\left[\left(\mathrm{ut} / \mathrm{p}^{\mathrm{q}}\right)\right]^{\mathrm{m}}$

Comparing the coefficient of $u^{m} t^{n}$ on both sides of (9) we see that the coefficient of $u^{m} t^{n}$ when $n \neq m$, then the right hand member of (9) is zero and when $n=m$ then the right hand member is non zero.

\section{INTEGRAL REPRESENTATIONS}

\section{A. integral representation for $Y_{n}^{(\alpha)}(x, r, p, k)$}

Osler [5] has given a fractional derivative formula as:

$\mathrm{D}_{\mathrm{g}(\mathrm{x})}^{(\alpha)}\{\mathrm{f}(\mathrm{z})\}=\mathrm{D}_{\mathrm{h}(\mathrm{x})}^{(\alpha)}\left[\frac{\mathrm{f}(\mathrm{z}) \mathrm{g}(\mathrm{z})}{\mathrm{h}(\mathrm{z})}\left(\frac{\mathrm{h}(\mathrm{z})-\mathrm{h}(\mathrm{w})}{\mathrm{g}(\mathrm{z})-\mathrm{g}(\mathrm{w})}\right)^{\alpha+1}\right]_{\mathrm{w}=\mathrm{z}}$

where $\mathrm{D}_{\mathrm{g}(\mathrm{x})}^{(\alpha)}\{\mathrm{f}(\mathrm{z})\}$ denotes the fractional derivation of order $\alpha$ with respect to $\mathrm{g}(\mathrm{z})$.

For $\alpha=\mathrm{m} \quad$ and $\quad h(z)=z$, we have

$D_{g(x)}^{(\alpha)}\{f(z)\}=D^{m}\left[f(z) g(z)\left(\frac{z-w}{g(z)-y(w)}\right)^{m+1}\right]_{w=z} \quad \forall m \in\{0,12 \ldots \ldots \ldots .$.

For the relatively more familiar derivative of order m now from (10), we have

$Y_{n}^{(\alpha)}(x, r, p, k)=\frac{x^{-1+n k-\alpha}}{k^{n} n !} \exp \left(p x^{r}\right)\left(x^{k+1} D_{x}\right)^{n}\left\{x^{\alpha+1} \exp \left(-p x^{r}\right)\right\}$,

$=\frac{k x^{k-\alpha-1}}{n !} \exp \left(-p x^{r}\right)(D x)^{n}\left[x^{\alpha+k n} \exp \left(-p x^{r}\right)\left[\left(\frac{x-y}{x^{k}-y^{k}}\right)^{n+1}\right]\right]_{y=x}$

from (11), we get integral representation as :

$\mathrm{Y}_{\mathrm{n}}^{\alpha}(\mathrm{x}, \mathrm{r}, \mathrm{p} ; \mathrm{k})=\frac{\mathrm{kr}^{-\alpha-1+\mathrm{kn}}}{2 \pi \mathrm{i}} \int_{\mathrm{c}}\left[\frac{\exp \left\{\mathrm{p}\left(\mathrm{u}^{\mathrm{r}}-\mathrm{x}^{\mathrm{r}}\right)\right\}^{\alpha+k n}}{\left[\left(\mathrm{u}^{\mathrm{k}}-\mathrm{x}^{\mathrm{k}}\right)\right]^{\mathrm{n}+1}}\right] \mathrm{du}$

taking $\mathrm{u}=\mathrm{y}(1+\mathrm{t})$ and after simple manipulation, we get $Y_{n}^{\alpha}(x, r, p ; k)=\frac{k}{2 \pi i} D_{t}^{n}\left[\frac{(1+t)^{a+k n} \exp ^{\prime}\left(-p^{r}(1+t)^{r}-1\right\} t^{n+1}}{\left((1+t)^{k}-1\right)^{n+1}}\right]_{t=0}$

(13)

from (15), we easily write the integral representation for $\mathrm{Y}_{\mathrm{n}}^{\alpha}(\mathrm{x}, \mathrm{r}, \mathrm{p} ; \mathrm{k})$ as:

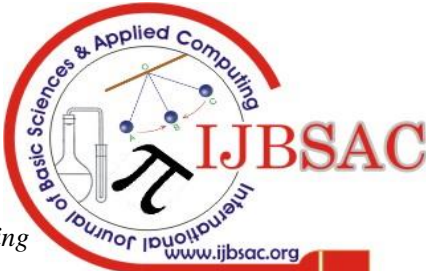


$Y_{n}^{\alpha}(x, r, p ; k)=\frac{k}{2 \pi i} \int_{c} \frac{(1+t)^{\alpha+k n} \exp \left\{-p^{r}(1+t)^{r}-1\right\}}{\left((1+t)^{k}-1\right)^{n+1}} d t$,

(14)

which $\mathrm{C}$ is a closed contour enclosing $\mathrm{t}=0$, but excluding $\mathrm{t}=1$ and the roots of the equation $(\mathrm{t}+1)^{\mathrm{k}}-1=0$

B. Integral representation for $Z_{n}^{\alpha}(x, r, p ; k)$ We consider,

$$
\int_{0}^{\infty} \exp \left(-\operatorname{ps}^{r} x^{r}\right) t^{\beta} Z_{n}^{\alpha}(x, r, p ; k) d t
$$

$=\frac{\Gamma\{(\alpha+1+\mathrm{kn}) / \mathrm{r}\}}{\mathrm{p}^{\mathrm{kn} / \mathrm{r}} \mathrm{n} !} \sum_{\mathrm{m}=0}^{\mathrm{n}}(-\mathrm{n})_{\mathrm{m}} \frac{\mathrm{p}^{\mathrm{km} / \mathrm{r}} \mathrm{x}^{\mathrm{km}}}{\mathrm{m} ! \Gamma\{(\alpha+1+\mathrm{km}) / \mathrm{r}\}} \int_{0}^{\infty} \mathrm{t}^{\beta+\mathrm{km}} \exp \left(-\mathrm{ps}^{\mathrm{r}} \mathrm{x}^{\mathrm{r}}\right) \mathrm{dt}$

$=\frac{\Gamma\{(\alpha+1+\mathrm{kn}) / \mathrm{r}\}}{\mathrm{p}^{\mathrm{kn}+\beta+1 / \mathrm{r}} \mathrm{r} \cdot \mathrm{n} ! \mathrm{s}^{1+\beta}} \sum_{\mathrm{m}=0}^{\mathrm{n}}(-\mathrm{n})_{\mathrm{m}} \frac{\Gamma\{(\mathrm{km}+\beta+1) / \mathrm{r}\}}{\mathrm{m} ! \Gamma\{(\alpha+1+\mathrm{km}) / \mathrm{r}\}}(\mathrm{x} / \mathrm{s})^{\mathrm{k}}$

(15)

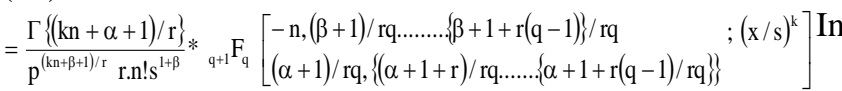
particular for $\alpha=\beta$, (15) reduces to the following form :

$\int_{0}^{\infty} \exp \left(-\mathrm{ps}^{\mathrm{r}} \mathrm{x}^{\mathrm{r}}\right) \mathrm{t}^{\alpha} \mathrm{Z}_{\mathrm{n}}^{(\alpha)}(\mathrm{x}, \mathrm{r}, \mathrm{p} ; \mathrm{k}) \mathrm{dt}=\frac{\Gamma((\alpha+1+\mathrm{kn}) / \mathrm{r}\}\left(\mathrm{s}^{\mathrm{k}}-\mathrm{x}^{\mathrm{k}}\right)^{\mathrm{n}}}{\mathrm{P}^{(\mathrm{kn}+\alpha+1) / \mathrm{r}} \mathrm{r} \cdot \mathrm{n} ! \mathrm{s}^{1+\alpha+\mathrm{k}_{\mathrm{n}}}} \mathrm{rn} \cdot \mathrm{s} \mathrm{s}^{1+\alpha+\mathrm{kn}}$

(16)

now applying inverse Laplace transform techniques to (16),we get the integral representation for $Z_{n}^{(\alpha)}(x, r, p ; k)$

$\frac{\mathrm{m} ! \mathrm{u}^{1+\alpha-\mathrm{r}}}{\Gamma\{(1+\alpha+\mathrm{kn}) / \mathrm{r}\}} \mathrm{Z}_{\mathrm{n}}^{(\alpha)}\left(\mathrm{u}^{1 / \mathrm{r}}, \mathrm{r}, \mathrm{p} ; \mathrm{k}\right)=\frac{1}{2 \pi \mathrm{i}} \int_{\mathrm{C}} \frac{\exp (\mathrm{ut})\left[(\mathrm{t} / \mathrm{p})^{\mathrm{k} / \mathrm{r}}-1\right]^{\mathrm{n}}}{\mathrm{t}^{\{(1+\alpha+\mathrm{kn}) / \mathrm{r}\}}} d t$

putting $\mathrm{u}=\mathrm{x}^{\mathrm{r}}$ and $\mathrm{t}=\mathrm{ps}^{\mathrm{r}}$ in (17), we get:

$\frac{\mathrm{n} ! \mathrm{p}^{(1+\alpha-\mathrm{r}+\mathrm{kn}) / \mathrm{r}} \mathrm{x}^{\alpha-\mathrm{r}+1}}{\Gamma\{(1+\alpha+\mathrm{kn}) / \mathrm{r}\}} \mathrm{Z}_{\mathrm{n}}^{(\alpha)}(\mathrm{x}, \mathrm{r}, \mathrm{p} ; \mathrm{k})=\frac{\mathrm{r}}{2 \pi \mathrm{i}} \int_{\mathrm{C}} \frac{\exp \left(\mathrm{ps}^{\mathrm{r}} \mathrm{x}^{\mathrm{r}}\right)\left[\mathrm{s}^{\mathrm{k}}-1\right]^{\mathrm{n}}}{\mathrm{s}^{(2+\alpha-\mathrm{r}+\mathrm{kn})}} \mathrm{ds}$

where $\mathrm{c}$ is contour enclosing $\mathrm{s}=0$ when $\alpha, \mathrm{r}, \mathrm{k}$ and $\mathrm{n}$ are integers.

We also have differential formula for $\mathrm{Z}_{\mathrm{n}}^{(\alpha)}(\mathrm{x}, \mathrm{r}, \mathrm{p} ; \mathrm{k})$ as

$\frac{\mathrm{p}^{(1+\alpha-r+k n) / r} \mathrm{x}^{\alpha-r+1}}{\Gamma\{(1+\alpha+\mathrm{kn}) / \mathrm{r}\}} \mathrm{Z}_{\mathrm{n}}^{(\alpha)}(\mathrm{x}, \mathrm{r}, \mathrm{p} ; \mathrm{k})=\frac{\mathrm{r}}{(1+\alpha-\mathrm{r}+\mathrm{kn}) !} \mathrm{D}_{\mathrm{s}}^{(1+\alpha-\mathrm{r}+\mathrm{knn}) !}\left[\frac{\exp \left(\mathrm{ps}^{\mathrm{r}} \mathrm{x}^{\mathrm{r}}\right)\left[\mathrm{s}^{\mathrm{k}}-1\right]^{\mathrm{n}}}{\mathrm{s}^{(2+\alpha-\mathrm{r}+\mathrm{knn})}}\right]_{\mathrm{s}=0}$

(19)

In particular, the above result reduces to the corresponding result Spencer and Feno (3) and Konhauser (5).

\section{RECURRENCE RELATIONS}

The polynomials $Y_{n}^{(\alpha)}(x, r, p ; k)$ and $Z_{n}^{(\alpha)}(x, r, p ; k)$ satisfy the recurrence relation

A. The recurrence relation for $Y_{n}^{(\alpha)}(x, r, p ; k)$ $\left(D_{x}-p x^{r-1}\right) Y_{n}^{(\alpha)}(x, r, p ; k)=\left(-p x^{r-1}\right) Y_{n}^{(\alpha+r)}(x, r, p ; k)$

$\left(\mathrm{p}^{-1} \mathbf{r}^{-1} \mathrm{X}^{1-\mathrm{r}} \mathbf{D}_{\mathrm{x}-1}\right) \mathbf{Y}_{\mathrm{n}}^{(\alpha)}(\mathrm{x}, \mathrm{r}, \mathrm{p} ; \mathrm{k})=-\mathrm{Y}_{\mathrm{n}}^{(\alpha+\mathrm{r})}(\mathrm{x}, \mathrm{r}, \mathrm{p} ; \mathrm{k})$

(21) $\left(\mathrm{p}^{-1} \mathrm{r}^{-1} \mathrm{x}^{1-\mathrm{r}} \mathrm{D}_{\mathrm{x}}-1\right)^{\mathrm{m}} \mathrm{Y}_{\mathrm{n}}^{(\alpha)}(\mathrm{x}, \mathrm{r}, \mathrm{p} ; \mathrm{k})=(-1)^{\mathrm{m}} \mathrm{Y}_{\mathrm{n}}^{(\alpha+\mathrm{mr})}(\mathrm{x}, \mathrm{r}, \mathrm{p} ; \mathrm{k})$

(22) $\left(-\mathrm{p}^{-1} \mathrm{r}^{-1} \mathrm{x}^{1-\mathrm{r}} \mathrm{D}_{\mathrm{x}}+1\right)^{\mathrm{q}} \mathrm{Y}_{\mathrm{n}}^{(\alpha)}(\mathrm{x}, \mathrm{r}, \mathrm{p} ; \mathrm{k})=\mathrm{Y}_{\mathrm{n}}^{(\alpha+\mathrm{k})}(\mathrm{x}, \mathrm{r}, \mathrm{p} ; \mathrm{k})$

(23)

where $\mathrm{k} / \mathrm{r}=\mathrm{q}$ is a positive integer. $\left[\left(-p^{-1} r^{-1} x^{1-r} D_{x}+1\right)^{q}-1\right] Y_{n}^{(\alpha)}(x, r, p ; k)=Y_{n-1}^{(\alpha+k)}(x, r, p ; k)$

$Y_{n}^{(\alpha+k)}(x, r, p ; k)-Y_{n}^{(\alpha)}(x, r, p ; k)=Y_{n-1}^{(\alpha+k)}(x, r, p ; k)$

$\left(\mathrm{xD}_{\mathrm{x}}+\alpha+\mathrm{kn}-\mathrm{prx}^{\mathrm{r}}\right) \mathrm{Y}_{\mathrm{n}}^{(\alpha)}(\mathrm{x}, \mathrm{r}, \mathrm{p} ; \mathrm{k})=\mathrm{k}(\mathrm{n}+1) \mathrm{Y}_{\mathrm{n}}^{(\alpha)}(\mathrm{x}, \mathrm{r}, \mathrm{p} ; \mathrm{k})$

$\left(\mathrm{xD}_{\mathrm{x}}+\alpha+1-\mathrm{k}-\operatorname{prx}^{\mathrm{r}}\right) \mathrm{Y}_{\mathrm{n}}^{(\alpha)}(\mathrm{x}, \mathrm{r}, \mathrm{p} ; \mathrm{k})=\mathrm{k}(\mathrm{n}+1) \mathrm{Y}_{\mathrm{n}+\mathrm{1}}^{(\alpha-\mathrm{k})}(\mathrm{x}, \mathrm{r}, \mathrm{p} ; \mathrm{k})$

$(\alpha+1-k) Y_{n}^{(\alpha)}(x, r, p ; k)=\operatorname{prx}^{r} Y_{n}^{(\alpha+r)}(x, r, p ; k)+k(n+1) Y_{n+1}^{(\alpha-k)}(x, r, p ; k)$

$k(n+1) Y_{n+1}^{(\alpha)}(x, r, p ; k)=(\alpha+k n+1) Y_{n}^{(\alpha)}(x, r, p ; k)-\operatorname{prx}^{r} Y_{n+1}^{(\alpha+r)}(x, r, p ; k)$

B. The recurrence relation for $Z_{N}^{(\alpha)}(x, r, p ; k)$

$\mathrm{D}_{\mathrm{x}} \mathrm{Z}_{\mathrm{n}}^{(\alpha)}(\mathrm{x}, \mathrm{r}, \mathrm{p} ; \mathrm{k})=-\mathrm{kx}^{\mathrm{k}-1} \mathrm{Z}_{\mathrm{n}-1}^{(\alpha+\mathrm{k})}(\mathrm{x}, \mathrm{r}, \mathrm{p} ; \mathrm{k})$

$\left(\mathrm{x}^{1-\mathrm{k}} \mathrm{D}_{\mathrm{x}}\right)^{\mathrm{m}} \mathrm{Z}_{\mathrm{n}}^{(\alpha)}(\mathrm{x}, \mathrm{r}, \mathrm{p} ; \mathrm{k})=(-\mathrm{k})^{\mathrm{m}} \mathrm{Z}_{\mathrm{n}-\mathrm{m}}^{(\alpha+\mathrm{m})}(\mathrm{x}, \mathrm{r}, \mathrm{p} ; \mathrm{k})$

$\left(\mathrm{xD}_{\mathrm{x}}-\mathrm{k}_{\mathrm{n}}\right) \mathrm{Z}_{\mathrm{n}}^{(\alpha)}(\mathrm{x}, \mathrm{r}, \mathrm{p} ; \mathrm{k})=\frac{\mathrm{kp}^{-\mathrm{k}} \Gamma\{(1+\alpha+\mathrm{kn}) / \mathrm{r}\}}{\mathrm{r} \Gamma\{(1+\alpha+\mathrm{k}(\mathrm{n}-1)) / \mathrm{r}\}} \mathrm{Z}_{\mathrm{n}-1}^{(\alpha)}(\mathrm{x}, \mathrm{r}, \mathrm{p} ; \mathrm{k})$

$\left(\left(\mathrm{xD}_{\mathrm{x}}+\alpha-\mathrm{r}\right)+1\right) \mathrm{Z}_{\mathrm{n}}^{(\alpha)}(\mathrm{x}, \mathrm{r}, \mathrm{p} ; \mathrm{k})=(1+\alpha-\mathrm{r}+\mathrm{kn}) \mathrm{Z}_{\mathrm{n}}^{(\alpha-\mathrm{r})}(\mathrm{x}, \mathrm{r}, \mathrm{p} ; \mathrm{k})$

$(1+\alpha-r+k n) Z_{n}^{(\alpha)}(x, r, p ; j)-(1+\alpha-r+k n) Z_{n}^{(\alpha-r)}(x, r, p ; k)=\frac{k p^{-1} \Gamma\{(1+\alpha+k n) / r\}}{r \Gamma\{(1+\alpha+k(n-1)) / r\}} Z_{n-1}^{(\alpha)}(x, r, p ; k)$

$\left[\left(p^{-1} r^{-1} x^{1-r} D_{x}\right)^{q}-1\right] x^{1+\alpha-r} Z_{n}^{(\alpha)}(x, r, p ; k)=(n+1) x^{1+\alpha-r-k(\alpha-k)} Z_{n+1}^{(\alpha)}(x, r, p ; k)$

where $\mathrm{q}=\mathrm{k} / \mathrm{r}$.

\section{MAIN RESULTS}

A. proofs from (20) to (31)

Rewrite equation (15) in the following form:

$\exp \left(-\mathrm{px}^{\mathrm{r}}\right) \mathrm{Y}_{\mathrm{n}}^{\alpha}\left(\mathrm{x}, \mathrm{r}, \mathrm{p} ; \mathrm{k}=\frac{\mathrm{k}}{2 \pi \mathrm{i}} \int_{\mathrm{C}}\left[\frac{(1+\mathrm{t})^{\alpha+\mathrm{kn}} \exp \left\{-\mathrm{px}^{\mathrm{r}}(1+\mathrm{t})^{\mathrm{r}}\right\}}{\left((1+\mathrm{t})^{\mathrm{k}}-1\right)^{\mathrm{n}+1}}\right]\right) \mathrm{dt}$

differentiating both sides with respect to $\mathrm{x}$, we get :

$\left(D_{x}-p^{r} x^{r-1}\right) Y_{n}^{\alpha}\left(x, r, p ; k=\frac{k\left(-p r x^{r-1}\right)}{2 \pi i} \int_{C}\left[\frac{(1+t)^{\alpha+r+k n} \exp \left\{-p x^{r}(1+t)^{r}\right\}}{\left((1+t)^{k}-1\right)^{n+1}}\right]\right) d t$

Using (26), we get :

$\left(D_{x}-p^{r x}{ }^{r-1}\right) Y_{n}^{(\alpha)}(x, r, p ; k)=\left(-p^{r}{ }^{r-1}\right) Y_{n}^{(\alpha+r)}(x, r, p ; k)$

which proves (19)

Now multiplying (19) both sides by $\mathrm{p}^{-1} \mathrm{r}^{-1} \mathrm{x}^{1-\mathrm{r}}$ and after simplification we get result (20)

result (21) and (22) are obvious iterations of (20).

- $\quad$ proof of (20)

Subtracting (23) both sides by $Y_{n}^{(\alpha)}(x, r, p ; k)$

and after some simplification we get:

$\left[\left(-p^{-1} r^{-1} x^{1-r} D_{x}+1\right)^{q}-1\right] \quad Y_{n}^{(\alpha)}(x, r, p ; k)=Y_{n-1}^{(\alpha+k)}(x, r, p ; k)$

- $\quad$ proof of (21)

From equation (22) and (23), we get :

$Y_{\mathrm{n}}^{(\alpha+\mathrm{k})}(\mathrm{x}, \mathrm{r}, \mathrm{p} ; \mathrm{k})-\mathrm{Y}_{\mathrm{n}}^{(\alpha)}(\mathrm{x}, \mathrm{r}, \mathrm{p} ; \mathrm{k})=\mathrm{Y}_{\mathrm{n}-1}^{(\alpha+\mathrm{k})}(\mathrm{x}, \mathrm{r}, \mathrm{p} ; \mathrm{k})$

\section{- $\quad$ proof of (22)}

Rewrite equation (15), in the following form :

$\exp \left(-\mathrm{px}^{\mathrm{r}}\right) \mathrm{Y}_{\mathrm{n}}^{\alpha}\left(\mathrm{x}, \mathrm{r}, \mathrm{p} ; \mathrm{k}=\frac{\mathrm{k}}{2 \pi \mathrm{i}} \int_{\mathrm{C}}\left[\frac{(\mathrm{x})^{-\alpha-1-\mathrm{kn}} \exp \left(-\mathrm{pu}^{\mathrm{r}}\right)(\mathrm{u})^{\alpha+\mathrm{kn}}}{\left((\mathrm{u}+\mathrm{x})^{\mathrm{k}}-1\right)^{\mathrm{n}+1}}\right]\right) \mathrm{du}$

differentiating both sides with respect to ' $x$ ' and after simplification we get : 


\section{Generalization of Konhauser Polynomials}

$\left(\mathrm{xD}_{\mathrm{x}}+\alpha+\mathrm{kn}-\mathrm{prx}^{\mathrm{r}}\right) \mathrm{Y}_{\mathrm{n}}^{(\alpha)}(\mathrm{x}, \mathrm{r}, \mathrm{p} ; \mathrm{k})=\mathrm{k}(\mathrm{n}+1) \mathrm{Y}_{\mathrm{n}}^{(\alpha)}(\mathrm{x}, \mathrm{r}, \mathrm{p} ; \mathrm{k})$

\section{- $\quad$ proof of (23)}

Rewrite equation (15), in the following form :

$\exp \left(-\mathrm{px}^{\mathrm{r}}\right) \mathrm{Y}_{\mathrm{n}}^{\alpha}\left(\mathrm{x}, \mathrm{r}, \mathrm{p} ; \mathrm{k}=\frac{\mathrm{k}}{2 \pi \mathrm{i}} \int_{\mathrm{C}}\left[\frac{\exp \left(-\mathrm{pu}^{\mathrm{r}}\right)(\mathrm{u} / \mathrm{x})^{\alpha+\mathrm{kn}}}{\left((\mathrm{u} / \mathrm{x})^{\mathrm{k}}-1\right)^{\mathrm{n}+1}}\right]\right) \mathrm{du}$

differentiating both sides with respect to $\mathrm{x}$ and rearranging terms, we get

$\left(\mathrm{xD}_{\mathrm{x}}+\alpha+1-\mathrm{k}-\mathrm{prx}^{\mathrm{r}}\right) \mathrm{Y}_{\mathrm{n}}^{(\alpha)}(\mathrm{x}, \mathrm{r}, \mathrm{p} ; \mathrm{k})=\mathrm{k}(\mathrm{n}+1) \mathrm{Y}_{\mathrm{n}+1}^{(\alpha-\mathrm{k})}(\mathrm{x}, \mathrm{r}, \mathrm{p} ; \mathrm{k})$ which proves (26).

- $\quad$ proof of (24)

Eliminating the term $\mathrm{xD}_{\mathrm{x}} \mathrm{Y}_{\mathrm{n}}^{(\alpha)}(\mathrm{x}, \mathrm{r}, \mathrm{p} ; \mathrm{k})$ between (21) and (23) we get:

$(\alpha+1-k) Y_{n}^{(\alpha)}(x, r, p ; k)-\operatorname{prx}^{r} Y_{n}^{(\alpha+r)}(x, r, p ; k)=k(n+1) Y_{n+1}^{(\alpha-k)}(x, r, p ; k)$

on transposition we get :

$(\alpha+1-k) Y_{n}^{(\alpha)}(x, r, p ; k)-\operatorname{prx}^{r} Y_{n}^{(\alpha+r)}(x, r, p ; k)=k(n+1) Y_{n+1}^{(\alpha-k)}(x, r, p ; k)$

- $\quad$ proof of (25)

Eliminating the term $\mathrm{xD}_{\mathrm{x}} \mathrm{Y}_{\mathrm{n}}^{(\alpha)}(\mathrm{x}, \mathrm{r}, \mathrm{p} ; \mathrm{k})$ between (21) and (26), we get:

$(\alpha+k n+1) Y_{n}^{(\alpha)}(x, r, p ; k)-p r x^{r} Y_{n+1}^{(\alpha+r)}(x, r, p ; k)=k(n+1) Y_{n+1}^{(\alpha+k)}(x, r, p ; k)$

- $\quad$ proof of (26)

Now putting $\mathrm{u}=\mathrm{Sx}$ in relation (19), we get:

$\frac{\mathrm{n} ! \mathrm{p}^{(1+\alpha-\mathrm{r}+\mathrm{kn}) / \mathrm{r}}}{\Gamma\{(1+\alpha+\mathrm{kn}) / \mathrm{r}\}} \mathrm{Z}_{\mathrm{n}}^{(\alpha)}(\mathrm{x}, \mathrm{r}, \mathrm{p} ; \mathrm{k})=\frac{\mathrm{r}}{2 \pi \mathrm{i}} \int_{\mathrm{C}} \frac{\exp \left(\mathrm{pu}^{\mathrm{r}}\right)\left[\mathrm{u}^{\mathrm{k}}-\mathrm{x}^{\mathrm{k}}\right]^{\mathrm{n}}}{\mathrm{u}^{(2+\alpha-\mathrm{r}+\mathrm{kn}) / \mathrm{r}}} \mathrm{du}$

differentiating both the sides with respect to $\mathrm{x}$ and after simplification we get:

$\frac{\mathrm{n} ! \mathrm{p}^{(1+\alpha-\mathrm{r}+\mathrm{kn}) / \mathrm{r}}}{\Gamma\{(1+\alpha+\mathrm{kn}) / \mathrm{r}\}} \mathrm{D}_{\mathrm{x}}\left[\mathrm{Z}_{\mathrm{n}}^{(\alpha)}(\mathrm{x}, \mathrm{r}, \mathrm{p} ; \mathrm{k})\right]=\frac{\mathrm{mnkx} \mathrm{k}^{-\mathrm{k}-1}}{2 \pi \mathrm{i}} \int_{\mathrm{C}} \frac{\exp \left(\mathrm{pu}^{\mathrm{r}}\right)\left[\mathrm{u}^{\mathrm{k}}-\mathrm{x}^{\mathrm{k}}\right]^{\mathrm{n}-1}}{\mathrm{u}^{(2+\alpha-\mathrm{r}+\mathrm{k}(\mathrm{n}-1)) / \mathrm{r}}} \mathrm{du}$

after some simplification we get

$\mathrm{D}_{\mathrm{x}} \mathrm{Z}_{\mathrm{n}}^{(\alpha)}(\mathrm{x}, \mathrm{r}, \mathrm{p} ; \mathrm{k})=-\mathrm{kx}^{\mathrm{k}-1} \mathrm{Z}_{\mathrm{n}-\mathrm{1}}^{(\alpha+\mathrm{k})}(\mathrm{x}, \mathrm{r}, \mathrm{p} ; \mathrm{k})$

\section{- $\quad$ proof of (27)}

From equation (31), we have

$\mathrm{x}^{1-\mathrm{k}} \mathrm{D}_{\mathrm{x}} \mathrm{Z}_{\mathrm{n}}^{(\alpha)}(\mathrm{x}, \mathrm{r}, \mathrm{p} ; \mathrm{k})=-\mathrm{k} \mathrm{Z}_{\mathrm{n}-1}^{(\alpha+\mathrm{k})}(\mathrm{x}, \mathrm{r}, \mathrm{p} ; \mathrm{k})$

which on integration ' $\mathrm{n}$ ' times further gives the recurrence relation:

$$
\left(x^{1-k} D_{k}\right)^{m} Z_{n}^{(\alpha)}(x, r, p ; k)=(-k)^{m} Z_{n-m}^{(\alpha+k m)}(x, r, p ; k)
$$

- $\quad$ proof of (28)

Now putting $\mathrm{u}=\mathrm{sx}$ in relation (18), we get :

$\frac{n ! p^{(1+\alpha-r+k n) / r}}{\Gamma\{(1+\alpha+k n) / r\}} x^{-k n} Z_{n}^{(\alpha)}(x, r, p ; k)=\frac{r}{2 \pi i} \int_{C} \frac{\exp \left(\mathrm{pu}^{\mathrm{r}}\right)\left[(\mathrm{u} / \mathrm{x})^{\mathrm{k}}-1\right]^{\mathrm{n}}}{\mathrm{u}^{(2+\alpha-\mathrm{r}+\mathrm{kn}) / \mathrm{r}}} \mathrm{du}$

differentiating both the sides with respect to $\mathrm{x}$ and after simplification we get

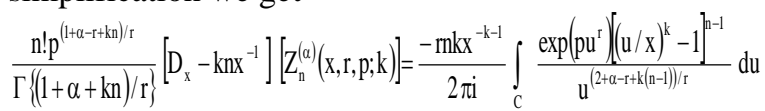

or

$\left(x D_{x}-k n\right) Z_{n}^{(\alpha)}(x, r, p ; k)=\frac{-k p^{-k} \Gamma\{(1+\alpha+k n) / r\}}{r \Gamma\{(1+\alpha+k(n-1)) / r)\}} Z_{n-1}^{(\alpha)}(x, r, p ; k)$

- $\quad$ proof of (29)
Differentiating (19), both the sides with respect to $\mathrm{x}$ we get : $\left(\mathrm{xD}_{\mathrm{x}}+\alpha-\mathrm{r}+1\right) \mathrm{Z}_{\mathrm{n}}^{(\alpha)}(\mathrm{x}, \mathrm{r}, \mathrm{p} ; \mathrm{k})=\frac{\mathrm{r} \Gamma[(1+\alpha+\mathrm{kn}) / \mathrm{r}]}{\Gamma[(1+\alpha-\mathrm{r}+\mathrm{kn}) / \mathrm{r}]} \mathrm{Z}_{\mathrm{n}}^{(\alpha-\mathrm{r})}(\mathrm{x}, \mathrm{r}, \mathrm{p} ; \mathrm{k})$ or $\left(\left(x D_{x}+\alpha-r\right)+1\right) Z_{n}^{(\alpha)}(x, r, p ; k)=(1+\alpha-r+k n) Z_{n}^{(\alpha-r)}(x, r, p ; k)$

\section{- $\quad$ proof of (30)}

Eliminating the term $\mathrm{xD}_{\mathrm{x}} \mathrm{Z}_{\mathrm{n}}^{(\alpha)}(\mathrm{x}, \mathrm{r}, \mathrm{p} ; \mathrm{k})$ from (30) and (32), we obtain the following recurrence relation:

$(1+a-r+k n) Z_{n}^{(a)}(x, r, r, p ; k)-(1+a-r+k n) Z_{n}^{(a-r)}(x, r, p, p, k)=\frac{\left.k^{-1} p^{-1} \Gamma(1+a+k n) / r\right\}}{r \Gamma(1+a+k(n-1)) / r\}} Z_{n-1}^{(\alpha)}(x, r, p ;, k)$

- $\quad$ proofs of (31)

Rewrite the equation (10) in the following form :

$\frac{\mathrm{n} ! \mathrm{p}^{(1+\alpha-\mathrm{r}+\mathrm{kn}) / \mathrm{r}} \mathrm{x}^{\alpha-\mathrm{r}+1}}{\Gamma\{(1+\alpha+\mathrm{kn}) / \mathrm{r}\}} \mathrm{Z}_{\mathrm{n}}^{(\alpha)}(\mathrm{x}, \mathrm{r}, \mathrm{p} ; \mathrm{k})=\frac{\mathrm{r}}{2 \pi \mathrm{i}} \int_{\mathrm{C}} \frac{\exp \left(\mathrm{ps}^{\mathrm{r}} \mathrm{x}^{\mathrm{r}}\right) \mathrm{p}^{\mathrm{q}} \mathrm{s}^{\mathrm{k}}\left[\mathrm{s}^{\mathrm{k}}-1\right]^{\mathrm{n}}}{\mathrm{s}^{(2+\alpha-\mathrm{r}+\mathrm{kn})}} \mathrm{ds}$

where $\mathrm{k} / \mathrm{r}=\mathrm{q}$,

or $=\frac{\mathrm{rp}^{\mathrm{q}}}{2 \pi \mathrm{i}} \int_{\mathrm{C}} \frac{\exp \left(\mathrm{ps}^{\mathrm{r}} \mathrm{x}^{\mathrm{r}}\right)\left[\mathrm{s}^{\mathrm{k}}-1\right]^{\mathrm{n}}}{\mathrm{s}^{(2+\alpha-\mathrm{r}+\mathrm{kn})}} \mathrm{ds}+\frac{\mathrm{rp}^{\mathrm{q}}}{2 \pi \mathrm{i}} \int_{\mathrm{C}} \frac{\exp \left(\mathrm{ps}^{\mathrm{r}} \mathrm{x}^{\mathrm{r}}\right)\left[\mathrm{s}^{\mathrm{k}}-1\right]^{\mathrm{n}}}{\mathrm{s}^{(2+(\alpha-\mathrm{k})-\mathrm{r}+\mathrm{k}(\mathrm{n}+1))}} \mathrm{ds}$ differentiating both sides with respect to $\mathrm{x}, \mathrm{q}$ times, we get : $\left[\left(p^{-1} r^{-1} x^{1-r} D_{x}\right)^{9}-1\right] x^{1+\alpha-r} Z_{n}^{(\alpha)}(x, r, p ; k)=(n+1) x^{1+\alpha-r-k(\alpha-k)} Z_{n+1}^{(\alpha)}(x, r, p ; k)$

\section{SPECIAL CASES}

The following known special cases of (11) and (12) are

- $\quad$ spencer and fano polynomials

Taking, $\mathrm{k}=2$, $\mathrm{r}=1, \mathrm{p}=1$; we get:

$\mathrm{Z}_{1}^{(\Omega)}(\mathrm{x})=\gamma_{1}^{(\Omega)}(\mathrm{x}, 1,1,2)$ and $\mathrm{Z}_{1}^{(\Omega)}(\mathrm{x}, 1,1,2)=\gamma_{1}^{(\Omega)}(\mathrm{x})$

- konhauser polynomials

Taking, $r=1, p=1$; we get:

$\gamma_{\mathrm{n}}^{(\Omega)}(\mathrm{x}, \mathrm{k})=\gamma_{\mathrm{n}}^{(\Omega)}(\mathrm{x}, 1,1, \mathrm{k})$ and $\mathrm{Z}_{\mathrm{n}}^{(\alpha)}(\mathrm{x}, \mathrm{k})=\mathrm{Z}_{\mathrm{n}}^{(\alpha)}(\mathrm{x}, 1,1 \mathrm{k})$

- laguerre polynomials

Taking, $\mathrm{k}=1, \mathrm{r}=1, \mathrm{p}=1$, we get:

$\mathrm{L}_{\mathrm{n}}^{(\alpha)}(\mathrm{x})=\gamma_{\mathrm{n}}^{(\alpha)}(\mathrm{x}, 1,1,1)=Z_{\mathrm{n}}^{(\alpha)}(\mathrm{x}, 1,1,1)$

\section{- bessel polynomials}

Other than above (11) and (12) also give rise to biorthogonal polynomials sets associated with Bessel polynomials given below for $k=-1$ and $r=-1$, we get :

$\gamma_{\mathrm{n}}^{(\alpha)}(\mathrm{x},-1, \beta-1)=[(-1) / \mathrm{n} !](\beta / \mathrm{n})^{\mathrm{n}} \gamma_{\mathrm{n}}^{(\alpha)}(\mathrm{x}, \alpha+\beta-2 \mathrm{n}, \beta)$

where $\gamma_{\mathrm{n}}^{(\alpha)}(\mathrm{x}, \alpha, \beta)$ is generalized Bessel polynomials defined by

$\gamma_{n}(x,-1, \beta,-1)=\beta^{-n} x^{-\alpha+2} \exp (\beta / n) D^{n}\left[x^{\alpha-2+2 n} \exp (-\beta / n)\right]$

clearly the above polynomials satisfy the biorthogonal property,

$\int_{0}^{\infty} x^{\alpha} e^{-\frac{\beta}{x}}\left(\frac{\beta}{x}\right)^{n} \gamma_{n}(x, \alpha+\beta-2 n, \beta) q_{m}(x, \alpha+\beta, \beta) d x=\frac{(-1)^{n-1} n ! \Gamma(m-\alpha+1)}{m ! \beta^{m-\alpha+1}} \delta_{m, n}$

\section{BILINEAR AND BILATERAL GENERATING RELATIONS}

In the section, we have derived some generating function for

Published By:

Blue Eyes Intelligence Engineering

\& Sciences Publication

(C) Copyright: All rights reserved. 
$\mathrm{Y}_{\mathrm{n}}^{(\alpha)}(\mathrm{x}, \mathrm{r}, \mathrm{p}, \mathrm{k})$ and $\mathrm{Z}_{\mathrm{n}}^{(\alpha)}(\mathrm{x}, \mathrm{r}, \mathrm{p}, \mathrm{k})$.

Now in this section we shall adopt group theoretic method to obtain a new class of bilinear and bilateral generating relations associated with

$Y_{n}^{(\alpha)}(x, r, p, k)$ and $Z_{n}^{(\alpha)}(x, r, p, k)$ all the result derived here appear in the form of some theorem. We prove the following theorems with application.

\section{Theorem-1}

If there exists a generating relation of the form

$$
\begin{aligned}
& G(x, w)=\sum_{n=0}^{\infty} a_{n} n ! \gamma_{n}^{(\alpha)}(x, r, p, k) w^{n} \\
& \exp \left[p x^{r}\left\{1-(1-t)^{-\frac{r}{k}}\right\}\right](1-t)^{-(\alpha+1) / k} G\left[x(1-t)^{-1 / k}, t v(1-t)\right]
\end{aligned}
$$

Proof: Consider the linear partial differential operator $\Omega$ as follows

$\Omega=x y \frac{\partial}{\partial x}+k^{2} \frac{\partial}{\partial x}+\left(\alpha+1-\mathrm{rpx}^{\mathrm{r}}\right) \mathrm{y}$

Such that

$$
\Omega=\left[Y_{n}^{(\alpha)}(x, r, p, k) n ! y^{n}=k(n+1) y^{n+1} Y_{n+1}^{(\alpha)}(x, r, p, k)\right]
$$

Such that Hence, clearly $\Omega$ form a raising Lie-operator for the class of function $\gamma_{\mathrm{n}}^{(\alpha)}(\mathrm{x}, \mathrm{r}, \mathrm{p} ; \mathrm{k})$. The multiplier representation of this operator is given by $\exp \left((w \Omega) f(x, y)=\exp \left[\operatorname{px}^{r}\left\{1-(1-k w y)^{-r / k}\right\}\right]\right)(1-k w y)^{-(\alpha+1) / k}$ * $\mathrm{f}\left[\mathrm{x}(1-\mathrm{kwy})^{-1 / \mathrm{k}}, \mathrm{y}(1-\mathrm{kwy})^{-1}\right]$

Let us now consider the following generating relation; $\mathrm{G}(\mathrm{x}, \mathrm{w})=\sum_{\mathrm{n}=0}^{\infty} \mathrm{a}_{\mathrm{n}} \mathrm{n} ! \gamma_{\mathrm{n}}^{(\alpha)}(\mathrm{x}, \mathrm{r}, \mathrm{p} ; \mathrm{k}) \mathrm{w}^{\mathrm{n}}$

replacing $\mathrm{w}$ by wyz in (37), we get;

$$
\mathrm{G}(\mathrm{x}, \mathrm{wyz})=\sum_{\mathrm{n}=0}^{\infty} \mathrm{a}_{\mathrm{n}} \mathrm{n} ! \gamma_{\mathrm{n}}^{(\alpha)}(\mathrm{x}, \mathrm{r}, \mathrm{p} ; \mathrm{k})(\mathrm{wyz})^{\mathrm{n}}
$$

operating both side of (38) by $\exp (\mathrm{w} \Omega$ ), we get; $\exp (w \Omega) G(x, w y z)=\exp (w \Omega), \sum_{n=0}^{\infty} a_{n} n ! \gamma_{n}^{(\alpha)}(x, r, p ; k)(w y z)^{n}$

(40)

now, using (37) the left hand member of (39) becomes.

$\exp \left[\mathrm{px}^{\mathrm{r}}\left\{1-(1-\mathrm{kwy})^{\mathrm{r} / \mathrm{k}}\right\}\right](1-\mathrm{kwy})^{-(\alpha+1) / \mathrm{k}}$

$\mathrm{G}\left[\mathrm{x}(1-\mathrm{kxy})^{-1 / \mathrm{k}}, \mathrm{y}(1-\mathrm{kwy})^{-1}\right]$

also using (34) the right hand member of (38) becomes

$\sum_{\mathrm{n}=0}^{\infty} \sum_{\mathrm{m}=0}^{\infty} \mathrm{a}_{\mathrm{n}} \mathrm{w}^{\mathrm{n}+\mathrm{m}} \mathrm{z}^{\mathrm{n}}(1 / \mathrm{m} !) \Omega^{\mathrm{m}}\left[\gamma_{\mathrm{n}}^{(\alpha)}(\mathrm{x}, \mathrm{r}, \mathrm{p}, \mathrm{k}) \mathrm{y}^{\mathrm{n}} \mathrm{n} !\right]$

$=\sum_{\mathrm{n}=0}^{\infty} \sum_{\mathrm{m}=0}^{\infty} \mathrm{k}^{\mathrm{m}} \mathrm{a}_{\mathrm{n}}(1 / \mathrm{m} !)(\mathrm{n}+\mathrm{m}) ! \mathrm{w}^{\mathrm{n}+\mathrm{m}} \mathrm{z}^{\mathrm{n}} \mathrm{y}^{\mathrm{n}+\mathrm{m}}\left[\gamma_{\mathrm{n}}^{(\alpha)}(\mathrm{x}, \mathrm{r}, \mathrm{p}, \mathrm{k})\right]$

$$
=\sum_{\mathrm{n}=0}^{\infty} \sum_{\mathrm{m}=0}^{\infty} \mathrm{a}_{\mathrm{n}-\mathrm{m}}(1 / \mathrm{m} !) \mathrm{n} !(\mathrm{kwy})^{\mathrm{n}-\mathrm{m}}\left[\gamma_{\mathrm{n}}^{(\alpha)}(\mathrm{x}, \mathrm{r}, \mathrm{p}, \mathrm{k})\right]
$$

equating (41) and (42) and then putting $\mathrm{kwy}=\mathrm{t}, \mathrm{zt} / \mathrm{k}=\mathrm{v}$, we get the following relation;

$\exp \left[\mathrm{px}^{\mathrm{r}}\left\{1-(1-\mathrm{t})^{-\mathrm{r} / \mathrm{k}}\right\}\right](1-\mathrm{t})^{-(\alpha+1) / \mathrm{k}} \mathrm{G}\left[\mathrm{x}(1-\mathrm{t})^{-1 / \mathrm{k}}, \mathrm{tv}(1-\mathrm{t})\right]$ $=\sum_{\mathrm{n}=0}^{\infty} \gamma_{\mathrm{n}}^{(\alpha)}(\mathrm{x}, \mathrm{r}, \mathrm{p} ; \mathrm{k}) \mathrm{t}^{\mathrm{n}} \sigma_{\mathrm{n}}(\mathrm{v})$

Where $\sigma_{\mathrm{n}}(\mathrm{v})=\sum_{\mathrm{n}=0}^{\infty} \mathrm{m} ! \mathrm{a}_{\mathrm{m}}\left(\begin{array}{l}\mathrm{n} \\ \mathrm{m}\end{array}\right) \mathrm{v}^{\mathrm{m}}$

This completes proof of the theorem.

\section{Theorem-2}

If there exists a bilinear generating relation of the form

$\mathrm{G}(\mathrm{x}, \mathrm{u}, \mathrm{w})=\sum_{\mathrm{n}=0}^{\infty} \mathrm{a}_{\mathrm{n}} \mathrm{w}^{\mathrm{n}}(\mathrm{n} !)^{2} \gamma_{\mathrm{n}}^{(\beta)}(\mathrm{u} ; \mathrm{s})$

then there exists a generating relation of the form :

$(1-\mathrm{tw})^{-1 / t}(1-\mathrm{sw})^{-(\beta+1) / s} \exp \left[\mathrm{px}^{\mathrm{r}}\left\{1-(1-\mathrm{wt})^{\mathrm{r} / \mathrm{t}}\right\}+\mathrm{u}^{\mathrm{r}}\{1-\mathrm{sw}\}^{-\mathrm{r} / \mathrm{s}}\right]$

$\mathrm{G}\left[\mathrm{x}(1-\mathrm{tw})^{-1 / \mathrm{t}}, \mathrm{u}(1-\mathrm{sw})^{-1 / \mathrm{s}}, \mathrm{wg}(1-\mathrm{wt})^{-1}(1-\mathrm{ws})^{-1}\right]$

$=\sum_{n=0}^{\infty} \sum_{j_{1}=0}^{\infty}(w g)^{m} n ! f_{n}^{(t, s)}(w, g, x) \gamma_{n}^{(\beta)}(u ; s)$

Where(44)

$$
\begin{aligned}
& \mathrm{f}_{\mathrm{n}}^{(\mathrm{t}, \mathrm{s})}(\mathrm{w}, \mathrm{g} ; \mathrm{x})=\sum_{\mathrm{j}_{2}=0}^{\min \left(\mathrm{n}, \mathrm{j}_{1}\right)} \frac{\mathrm{a}_{\mathrm{n}-\mathrm{j} 2}(w \mathrm{t})^{\mathrm{j}_{1}-\mathrm{j}_{2}} \mathrm{~s}^{\mathrm{j} 2} \mathrm{~g}^{\mathrm{j} 2}}{\left(\mathrm{j}_{1}-\mathrm{j}_{2}\right) !\left(\mathrm{j}_{2}\right) !} \\
& *\left(\mathrm{n}+\mathrm{j}_{1}-2 \mathrm{j}_{2}\right) ! \gamma_{\mathrm{n}+\mathrm{j}_{1}-2 / 2}^{(\alpha)}(\mathrm{x} ; \mathrm{t})
\end{aligned}
$$

\section{Proof:}

Consider the linear partial differential operator $\Omega_{\mathrm{i}}$ follows $\Omega_{\mathrm{i}}=\mathrm{y}_{\mathrm{i}}\left(\mathrm{x} \partial / \partial \mathrm{x}+\mathrm{ky}, \partial / \partial \mathrm{y}_{\mathrm{i}}+\alpha+1-\mathrm{rpx}^{\mathrm{r}}\right), \mathrm{i}=1,2$

Such that

(45) $\Omega\left[\gamma_{\mathrm{n}}^{(\alpha)}(\mathrm{x}, \mathrm{t}) \mathrm{n} ! \mathrm{y}_{\mathrm{i}}\right]^{\mathrm{n}}=\mathrm{k}(\mathrm{n}+1) ! \mathrm{y}_{\mathrm{i}}^{\mathrm{n}+1} \gamma_{\mathrm{n}+1}^{(\alpha)}(\mathrm{x}, \mathrm{t})$ hence, clearly $\Omega_{\mathrm{i}}$ form a raising Lie-operator for the class of function $\gamma_{\mathrm{n}}^{(\alpha)}(\mathrm{x}, \mathrm{r}, \mathrm{p} ; \mathrm{k})$. The multiplier representation of this operator is given by

$\exp \left(w \Omega_{i}\right) f\left(x, y_{i}\right)=\left(1-k_{w} y_{i}\right)^{-(\alpha+1) / k}$

$\exp \left[\operatorname{px}^{r}\left\{1-\left(1-k w y_{i}\right)^{-r / k}\right)\right] f\left[x\left(1-k w y_{i}\right)^{-1 / k}, y_{i}\left(1-k w y_{i}\right)^{-1}\right]$

assuming, that (46) exists, we substitute $\mathrm{wy}_{1} \mathrm{y}_{2} \mathrm{~g}$ in the place of $\mathrm{w}$ and operating both sides by exp $\left(w \Omega_{1}\right) \exp \left(w \Omega_{2}\right)$,

we get $\exp \left(w \Omega_{1}\right) \exp \left(w \Omega_{2}\right) G\left(x, u \cdot w y_{1} y_{2} g\right)$

$=\exp \left(w \Omega_{1}\right) \exp \left(w \Omega_{2}\right) \sum_{n=0}^{\infty} a_{n}\left(w y_{1} y_{2} g\right)^{n}(n !) \gamma_{n}^{(\alpha)}(x, t) \gamma_{n}^{(\beta)}(u ; s)$

now, using (36), the left hand member of (37) becomes $\exp \left(\mathrm{w}_{1}\right)\left[\left(1-\mathrm{swy}_{2}\right)^{-(\beta+1) / \mathrm{s}} \exp \left[\mathrm{pu}^{\mathrm{r}}\left\{1-\left(1-\mathrm{swy}_{2}\right)^{-\mathrm{r} / \mathrm{s}}\right\}\right]\right.$

$\mathrm{G}\left[\mathrm{u}\left(1-\mathrm{swy}_{2}\right)^{-1 / \mathrm{s}}, \mathrm{y}_{2}\left(1-\mathrm{swy}_{2}\right)^{-1}\right]$

$=\left(1-\mathrm{twy}_{1}\right)^{-(\alpha+1) / \mathrm{t}}\left(1-\mathrm{swy}_{2}\right)^{-(\beta+1) / \mathrm{s}}$

* exp $\left[\operatorname{px}^{\mathrm{r}}\left\{1-\left(1-\mathrm{kwy}_{\mathrm{i}}\right)^{-\mathrm{r} / \mathrm{t}}\right\}+\operatorname{pu}^{\mathrm{r}}\left\{1-\left(1-\mathrm{swy}_{2}\right)^{-\mathrm{r} / \mathrm{s}}\right\}\right]$

$\mathrm{G}\left[\mathrm{x}\left(1-\mathrm{twy}_{1}\right)^{-1 / \mathrm{k}}, \mathrm{u}\left(1-\mathrm{swy}_{2}\right)^{-1 / \mathrm{s}}, \mathrm{wy}_{1} \mathrm{y}_{2} \mathrm{~g}\left(1-\mathrm{twy}_{1}\right)^{-1}\left(1-\mathrm{swy}_{2}\right)^{-1}\right]$

also using (36), we see that the right member side of (37) becomes,

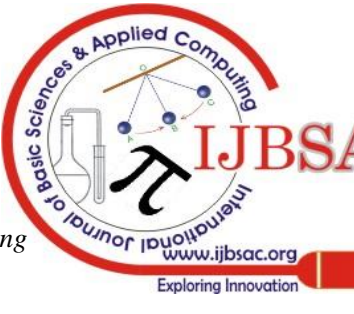




\section{Generalization of Konhauser Polynomials}

$\sum_{\mathrm{n}=0}^{\infty} \sum_{\mathrm{j}_{1}=0}^{\infty} \sum_{\mathrm{j}_{2}=0}^{\infty} \frac{\mathrm{a}_{\mathrm{n}}\left(w_{y_{1}} \mathrm{y}_{2} \mathrm{~g}\right)^{\mathrm{n}}(\mathrm{n} !)^{2} \Omega^{\mathrm{j}_{1}} \mathrm{w}^{\mathrm{j}_{2}} \gamma_{\mathrm{n}}^{(\beta)}(\mathrm{x} ; \mathrm{t}) \gamma_{\mathrm{n}}^{(\beta)}(\mathrm{u}, \mathrm{s})}{\mathrm{j}_{\mathrm{i}} ! \mathrm{j}_{2} !}$

$\sum_{n=0}^{\infty} \sum_{j_{1}=0}^{\infty} \sum_{j_{2}=0}^{\infty} \frac{a_{n} w^{n+j_{i}+j_{2}} g^{n}\left\{\Omega_{1}^{j_{1}}\left(\gamma_{n}^{(\alpha)}(x, t) n ! w y_{1}^{n}\right)\right\}\left\{\Omega_{2}^{j_{2}}\left(\gamma_{n}^{(\beta)}(u, s) n ! y_{2}^{n}\right)\right\}}{j_{i} ! j_{2} !}$

$\left.\sum_{n=0}^{\infty} \sum_{j_{1}=0}^{\infty} \sum_{j_{2}=0}^{\infty} \frac{a_{n} w^{n+j_{i}+j_{2}} g^{n}}{j_{i} ! j_{2} !}\left\{j^{j_{1}} \gamma_{n+j_{1}}^{(\alpha)}(x, t)\left(n+j_{1}\right)^{1+j_{1}} y_{1}\right\}\right)\left(s^{j_{2}} \gamma_{n+j_{2}}^{(\alpha)}(u, s)\left(n+j_{2}\right) ! n+j_{1} y_{2}\right\}$

$=\sum_{n=0}^{\infty} \sum_{j_{1}=0}^{\infty} \sum_{j_{2}=0}^{\min \left(n-j_{1}\right)} \frac{w^{n+j_{1}+j_{2}} g^{n-j_{2}}}{\left(j_{1}-j_{2}\right) j_{2} !} t^{j_{1}-j_{2}} \gamma_{n+j_{1}-j_{2}}^{(\alpha)}(u, t)\left(n+j_{1}-2 j_{2}\right) ! s^{j_{2}}$

$* \gamma_{\mathrm{n}}^{(\alpha)}(\mathrm{u}, \mathrm{s}) \mathrm{n} ! \mathrm{y}_{1}^{\mathrm{n}+\mathrm{j}_{1}-2 \mathrm{j}_{2}} \mathrm{y}_{2}^{\mathrm{n}}$

\section{Theorem-3}

If there exists a generating relation of the form

$G(x, w)=\sum_{n=0}^{\infty} a_{n} Z_{n}^{(\alpha)}(x, r, p ; k) w^{n}$

then there exists a generating relation of the form ;

$\left[\mathrm{rw}^{-1}(-\mathrm{vt} / \mathrm{wr})^{1 / \mathrm{r}}\right]^{(1 / \mathrm{r})-1}(-\mathrm{vt} / \mathrm{wr}) \mathrm{G}\left[\frac{\mathrm{x}\left(\mathrm{r}^{2} \mathrm{w}^{2}-\mathrm{vt}\right)^{1 / \mathrm{r}}}{(-\mathrm{vt})^{1 / \mathrm{r}}}, \frac{\mathrm{x}\left(\mathrm{r}^{2} \mathrm{w}^{2}-\mathrm{vt}\right)^{1 / \mathrm{r}}}{\mathrm{wr}}\right]$

$=\sum_{\mathrm{m}=0}^{\mathrm{n}} \mathrm{t}^{\mathrm{n}} \sigma_{\mathrm{n}}(\mathrm{x}, \mathrm{v})$

where

$\sigma_{\mathrm{n}}(\mathrm{x}, \mathrm{v})=\sum_{\mathrm{m}=0}^{\mathrm{n}}\left(\mathrm{a}_{\mathrm{m}} / \mathrm{m} !\right)(1-\{1+\alpha+\mathrm{km}\} / \mathrm{r})_{\mathrm{m}} \mathrm{Z}_{\mathrm{m}}^{(\alpha-\mathrm{nm})}(\mathrm{x}, \mathrm{r}, \mathrm{p} ; \mathrm{k}) \mathrm{v}^{\mathrm{m}}$

Proof:

Consider the linear partial differential operator $\Delta$ as follows :

$$
\Delta=y^{-r}\left(x \frac{\partial}{\partial x}+y \frac{\partial}{\partial y}-r+1\right) \text { Such that }
$$

$\Delta\left[\mathrm{Z}_{\mathrm{n}}^{(\alpha)}(\mathrm{x}, \mathrm{r}, \mathrm{p}, \mathrm{k}) \mathrm{y}^{\alpha}\right]=(1+\alpha-\mathrm{r}+\mathrm{kn}) \mathrm{Z}_{\mathrm{n}}^{(\alpha)}(\mathrm{x}, \mathrm{r}, \mathrm{p}, \mathrm{k})$

Hence, clearly $\Delta$ forms a raising Lie-operator for the class of function $Z_{n}^{(\alpha)}(x, r, p, k)$. The multiplier representation of this operator is given by

$\exp (w \Delta) f(x, y)=,\left(r w+y^{r}\right)^{(1-r) / r} y^{r} f\left[\left\{x\left(w r+y^{r}\right)^{1 / r}\right\} / y,\left(w r+y^{r}\right)^{1 / r}\right]$

(53)

Now consider the following generating relation;

$\mathrm{G}(\mathrm{x}, \mathrm{w})=\sum_{\mathrm{n}=0}^{\infty} \mathrm{a}_{\mathrm{n}} \mathrm{Z}_{\mathrm{n}}^{(\alpha)}(\mathrm{x}, \mathrm{r}, \mathrm{p}, \mathrm{k}) \mathrm{w}^{\mathrm{n}}$

replacing $w$ by wz and then multiplying both sides of (54)

by $\mathrm{y}^{\alpha}$; we get

$\mathrm{G}(\mathrm{x}, \mathrm{wz}) \mathrm{y}^{\alpha}=\mathrm{y}^{\alpha} \sum_{\mathrm{n}=0}^{\infty} \mathrm{a}_{\mathrm{n}} \mathrm{Z}_{\mathrm{n}}^{(\alpha)}(\mathrm{x}, \mathrm{r}, \mathrm{p}, \mathrm{k})(\mathrm{wx})^{\mathrm{n}}$

operating both sides of (55) by exp (w $\Delta$ ), we get

$\exp (w \Delta)\left[G(x, w z) y^{\alpha}\right]=\exp (w \Delta)\left[y^{\alpha} \sum_{n=0}^{\alpha} a_{n} Z_{n}^{(\alpha)}(x, r, p, k)(w z)^{n}\right](56)$

now, using (51), the left hand member of (54), becomes:

$\left(r w+y^{r}\right)^{(1-r) / r} y^{\alpha+r} G\left[\left\{x\left(w r+y^{r}\right)^{1 / r}\right\} / y,\left(w r+y^{r}\right)^{1 / r}\right]$

(57)

also using (40), we see that the right hand member of (44) becomes

$\sum_{n=0}^{\infty} \sum_{m=0}^{\infty} a_{n} w^{n+m} z^{n} \Delta^{m}\left[z_{n}^{(\alpha)}(x, r, p ; k) y^{\alpha}\right]$

Published By:

Blue Eyes Intelligence Engineering

\& Sciences Publication

(C) Copyright: All rights reserved.
(58)

$$
\begin{gathered}
=y^{\alpha} \sum_{\mathrm{n}=0}^{\infty}(w z)^{\mathrm{n}} \sum_{\mathrm{m}=0}^{\mathrm{n}} \mathrm{a}_{\mathrm{n}-\mathrm{m}}(1 / \mathrm{m} !)(1-\{1+\alpha+\mathrm{k}(\mathrm{n}-\mathrm{m}) / \mathrm{r}\})_{\mathrm{m}} \mathrm{Z}_{\mathrm{n}-\mathrm{m}}^{(\alpha-\mathrm{mr})}(\mathrm{x}, \mathrm{r}, \mathrm{p} ; \mathrm{k}) \\
*\left(-1 / \mathrm{zy}^{\mathrm{t}}\right)^{\mathrm{m}}
\end{gathered}
$$

equation (45) and (46), we get;

$$
\begin{gathered}
\left(r w+y^{r}\right)^{(1-r) / r} y^{r} G\left[\left\{x\left(w r+y^{r}\right)^{1 / r}\right\} / y,\left(w r+y^{r}\right)^{1 / r}\right] \\
=\sum_{n=0}^{\infty}(w z)^{n} \sum_{m=0}^{n} a_{n-m}(1 / m !)(1-\{1+\alpha+k(n-m)\} / r)_{m} z_{n-m}^{(\alpha-m r)}(x, r, p ; k) \\
*\left(-r / z y^{t}\right)^{m}
\end{gathered}
$$

finally putting $\mathrm{wz}=\mathrm{t}$ and $-\mathrm{r} / \mathrm{z} \mathrm{y}^{\mathrm{t}}=\mathrm{v}$ in (59), we get

$$
\begin{gathered}
;\left[\mathrm{rw}+(-\mathrm{vt} / \mathrm{wr})^{1 / \mathrm{r}}\right]^{(1 / \mathrm{r})-\mathrm{k}}(-\mathrm{vt} / \mathrm{wr}) \mathrm{G}\left[\frac{\mathrm{x}\left(\mathrm{r}^{2} \mathrm{w}^{2}-\mathrm{vt}\right)^{1 / \mathrm{t}}}{(-\mathrm{vt})^{1 / \mathrm{r}}}, \frac{\left(\mathrm{r}^{2} \mathrm{w}^{2}-\mathrm{vt}\right)^{1 / \mathrm{r}}}{\mathrm{wr}}\right] \\
=\sum_{\mathrm{n}=0}^{\infty} \mathrm{t}^{\mathrm{n}} \sigma_{\mathrm{n}}(\mathrm{x}, \mathrm{v})
\end{gathered}
$$

where $_{\sigma_{\mathrm{n}}}(\mathrm{x}, \mathrm{v})=\sum_{\mathrm{m}=0}^{\mathrm{n}}\left(\mathrm{a}_{\mathrm{m}} / \mathrm{m} !\right)(1-\{1+\alpha+\mathrm{km}\} / \mathrm{r}) \mathrm{Z}_{\mathrm{m}}^{(\alpha-\mathrm{mr})}(\mathrm{x}, \mathrm{r}, \mathrm{p} ; \mathrm{k}) \mathrm{v}^{\mathrm{m}}$

This completes proof of the Theorem.

\section{Theorem-4}

$\mathrm{F}(\mathrm{x}, \mathrm{w})=\sum_{\mathrm{n}=0}^{\infty} \mathrm{a}_{\mathrm{n}} \gamma_{\mathrm{n}}^{(\alpha)}(\mathrm{x}, \mathrm{r}, \mathrm{p} ; \mathrm{k}) \mathrm{w}^{\mathrm{n}}$

then there exists a generating relation of the form,

$\exp ($ ut $) F\left[\left\{(- \text { ut } / p)+x^{1}\right\}^{1 / r}, t\right]=\sum_{n=0}^{\infty} t^{n} \sigma_{n}(x, u)$

Where

$$
\sigma_{n}(x, u)=\sum_{j=0}^{n} a_{n}(1 / j !) \gamma_{n}^{(\alpha+r j)}(x, r, p ; k) u^{j}
$$

Proof:

Consider the linear partial differential operator $\phi$ as follow

Such that $\phi=\mathrm{y}^{\mathrm{r}}\left(\mathrm{x}^{-\mathrm{r}+1} \partial / \partial \mathrm{x}-\mathrm{pr}\right)$

$\varphi=\left[\gamma_{\mathrm{n}}^{(\alpha)}(\mathrm{x}, \mathrm{r}, \mathrm{p} ; \mathrm{k}) \mathrm{y}^{\alpha}\right]=-\operatorname{pr} \gamma_{\mathrm{n}}^{(\alpha+\mathrm{r})}(\mathrm{x}, \mathrm{r}, \mathrm{p} ; \mathrm{k}) \mathrm{y}^{\alpha+\mathrm{r}}$ the multiplier representation of this operator is given by $\exp (w \phi) f(x, y)=\exp \left(-\operatorname{pry}^{r} w\right) f\left[\left(r y^{r} w+x^{r}\right)^{1 / r}, y\right]$ let us now consider the following generating relation

$\mathrm{F}(\mathrm{x}, \mathrm{w})=\sum_{\mathrm{n}=0}^{\infty} \mathrm{a}_{\mathrm{n}} \gamma_{\mathrm{n}}^{(\alpha)}(\mathrm{x}, \mathrm{r}, \mathrm{p} ; \mathrm{k}) \mathrm{w}^{\mathrm{n}}$

replacing $\mathrm{w}$ by $\mathrm{wz}$ and then multiplying both side of (49) by $\gamma^{\alpha}$, we get

$\gamma^{\alpha} F(x, w z)=\gamma^{\alpha} \sum_{n=0}^{\infty} a_{n} \gamma_{n}^{(\alpha)}(x, r, p ; k)(w z)^{n}$

operating both sides of (49) by exp ( $\mathrm{w} \phi)$, we get: $\exp (w \phi)\left[y^{\alpha} F(x, w z)\right]=\exp (w \phi)\left[\gamma^{\alpha} \sum_{n=0}^{\infty} a_{n} \gamma_{n}^{(\alpha)}(x, r, p ; k)(w z)^{n}\right]$ 
now using (47), we see that the left hand member of (50) becomes

$\exp \left(-\operatorname{pry}^{\mathrm{r}} \mathrm{w}\right) \gamma^{\alpha} \mathrm{F}\left[\left(\mathrm{ry}^{\mathrm{r}} \mathrm{w}+\mathrm{x}^{\mathrm{r}^{1 / \mathrm{r}}}\right), \mathrm{wz}\right]$

also using (46), the right hand member of (50) becomes,

$\sum_{n=0}^{\infty} \sum_{j=0}^{\infty} a_{n}(1 / j !) w^{n+j} z^{n} \phi^{j}\left[\gamma_{n}^{(\alpha)}(x, r, p ; k y) y^{\alpha}\right]$

$=\sum_{\mathrm{n}=0}^{\infty} \sum_{\mathrm{j}=0}^{\infty} \mathrm{a}_{\mathrm{n}-\mathrm{j}}(1 / \mathrm{j} !) \mathrm{w}^{\mathrm{n}} \mathrm{z}^{\mathrm{n}-\mathrm{j}}(-1)^{\mathrm{j}}(\mathrm{pr})^{\mathrm{j}}\left[\gamma_{\mathrm{n}}^{(\alpha+\mathrm{r})}(\mathrm{x}, \mathrm{r}, \mathrm{p} ; \mathrm{k}) \mathrm{y}^{\alpha+\mathrm{rj}}\right]$

(68) $=\sum_{n=0}^{\infty}(w t)^{n}\left[\sum_{j=0}^{\infty} a_{n-j}(1 / j !)\left(-\operatorname{rpy}^{r} / z\right)^{j}\left[\gamma_{n}^{(\alpha+r)}(x, r, p ; k) y^{\alpha}\right]\right]$

(69)

equating (51) and (52), we get:

$\exp \left(-\operatorname{pry}^{r} w\right) y^{\alpha} F\left[\left(\operatorname{ry}^{r} w+x^{r}\right)^{1 / r}, w z\right]$

$=\sum_{n=0}^{\infty}(w t)^{n}\left[\sum_{j=0}^{\infty} a_{n-j}(1 / j !)\left(-r p y^{r} / z\right)^{j}\left[\gamma_{n}^{(\alpha+r j)}(x, r, p ; k) y^{\alpha}\right]\right]$

finally, putting $\mathrm{wz}=\mathrm{t}$ and $\left(-\mathrm{rpy}^{\mathrm{r}} / \mathrm{z}\right)=\mathrm{u}$ in (53), we get $\exp ($ ut $) F\left[\left\{(- \text { ut } / P)+x^{r}\right\}^{1 / r}, t\right]=\sum_{n=0}^{\infty} t^{n} \sigma_{n}(x, u)$ where $\sigma_{\mathrm{n}}(\mathrm{x}, \mathrm{u})=\sum_{\mathrm{j}=0}^{\mathrm{n}} \mathrm{a}^{\mathrm{n}}(1 / \mathrm{j} !) \gamma_{\mathrm{n}}^{(\alpha+\mathrm{r})}(\mathrm{x}, \mathrm{r}, \mathrm{p}, \mathrm{k}) \mathrm{u}^{\mathrm{j}}$

This completes proof of the theorem

\section{CONCLUSION}

As we can see, the results are different in many conditions of generalization for one member of the pair of Konhauser biorthogonal polynomials, generating relation and its really get multiple representation of the such kind operators which are discuss in the above results.

\section{REFERENCES}

1. Didon, M.F.: "Sur certain system des polynomials associes Annales Sci. de," Ecole Normale sup. Tome 6, 1869, pp.111-125.

2. Deruyts, J. Bearux.: "Suriun class de polynomials conjuges Memories couounes memories de savat Etrangers Academic Royal des Science des letter" et des 1-Art-de belgiques Tome, 48., 1886

3. Spencer, L. And Fanno, O., "Penetration an diffusion of X-rays calculation of Special distribution by polynomials expansion,"J. Res. Nat. Bur. Standards, 46, 1951, pp. 446-461.

4. Presiser, S. : "An investigation of the biorthogonal polynomials desirable from ordinary differential equation of the third order,". J. Math. Anal. Appl. Vol. 4, 1962, PP. 38-64.

5. Konhauser, J.D.E.: "Biorthogonal polynomials suggested by the Laguerre polynomials”, Pacific, J. Math. 21, 1967, pp. 303-314.

6. Carlitz, L.: "A note on certain biorthogonal polynomials", Pacific , J. Math. 24, 1968 ,pp. 425-430.

7. Prabhakar, T.R. and Kashyap, N.K.,"Generating functions for the biorthogonal polynomials suggested by Special Jacobi Polynomicals,” J. Math. Sci. 14/15, 1979, pp. 21-26.

8. Rahman, M.,"A non negative representation of the unearization coefficient of the product of Jacobi polynomials," Can. J. Math. 33, 1981, pp.915-928.

9. Prabhakar, T.R. and Tomer, R.C.,"Biorthogonal polynomials suggested by the Legendre polynomials," J. Indian Math. Soc. (N.S.) 43, 1979, pp. 285-297.

10. Madhekar, H.C., and Thakre, N.K., "Biorthogonal polynomials suggested by the Jacobi polynomials",Pacific, J. Math. 100, 1982 pp. 417-424.

11. Al. Salam, W.A. and Verma, A.: "q-Analogues of some biorthogonal function",Canad. Math. Bull. 26, 1993, pp. 225-227.

12. Chandel, R.C.S.: "Generalized Laguerre polynomials and The polynomials related to them”, Ind. Jour. Maths. Vol. II, 2, 1969, PP. 57-66.

13. Osler, T.J.,"The fractional derivative of a composite Function", STAM, J. Math. Anal., 1970, pp.288-293.

Published By:

4. Srivastava, H.M. and Singhal, J.P., "A class of polynomials defined by generalized Rodrigues' formula,” Ann. Mat. Pura. Appl. (4), 4(90), 1971 pp. 75-85

15. Srivastava, H.M., "On the Konhauser sets of polynomials suggested by the Laguerre polynomials,"Pacific, J. Math. 49, 1973, pp.489-492.

16. Srivastava, P.N., "Classical polynomials a unified Presentation pub," inst. Math. (Beograd) (N.S.), 23(37), 1978, pp.169-177.

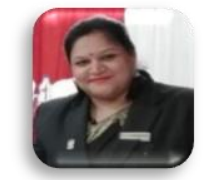

\section{AUTHORS PROFILE}

Mamta Dassani : has 15 years teaching experience and presently working as teaching assistant in the Department of Basic Science, Bundelkhand University, Jhansi, India. She received her Ph.D degree from Barkatullah University, Bhopal(M.P). she has presented many research papers in international meets. his research area is functional analysis.

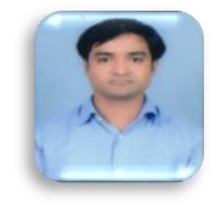

Mukesh kushwaha : has 7 years of teaching experience and presently working as teaching assistant in the Department of Basic Science, Bundelkhand University, Jhnasi, India. He has pulished many research papers in reputed journal and presented many research papers in international meets. his research interests are Fixed Point Theory, Fuzzy Optimization, Neural Network.
Blue Eyes Intelligence Engineering \& Sciences Publication

(C) Copyright: All rights reserved. 